\title{
La población en la reflexión jurídico-política de Jenofonte: el rol de los metecos atenienses en Póroi
}

\author{
Population in the political and legal thinking of Xenophon: the role of Athenian metics in the Póroi
}

\author{
Juan Bautista Bardi \\ Universidad de Buenos Aires, Argentina \\ juanbautistabardi@gmail.com \\ (iD https://orcid.org/0000-0002-1285-2070
}

\section{Resumen:}

\begin{abstract}
El presente artículo busca explicitar los presupuestos filosóficos que estarían cimentando el tratamiento que Jenofonte otorga a los metecos en los Póroi (II. 1-7). La forma en la que el filósofo ateniense decide incorporar a dicho grupo poblacional a su evaluación económica de la pólis, poniéndolos en la misma balanza que cualquier otro tipo de bien, respondería a una operación de objetivación calibrada por el concepto de "utilidad", la cual hunde sus raíces en la "teoría de la posesión” desarrollada al comienzo del Económico (I. 1-23).
\end{abstract}

Palabras clave: Jenofonte, Póroi, Metecos, Objetivación, Población, Derecho ático.

\section{ABSTRACT:}

The present study seeks to make explicit the philosophical foundations underlying Xenophon's treatment of metics in the Póroi (II. 1-5). The way in which the Athenian philosopher decides to incorporate that category of population in his economical assessment of the pólis, matching them with any other type of good, is the result of a process of objectivation structured by the concept of "utility" that is rooted in the "theory of property" developed at the beginning of the Oeconomicus (I. 1-23).

Keywords: Xenophon, Póroi, Metics, Objectivation, Population, Attic law.

\section{INTRODUCCIÓN ${ }^{1}$}

El objetivo de la siguiente investigación es el de explicitar los presupuestos filosóficos que estarían cimentando el tratamiento que Jenofonte otorga a los metecos en los Póroi (II. 1-7). Allí el filósofo ateniense presentará una serie de reformas legales y jurídicas que tendrán como uno de sus destinatarios predilectos este grupo poblacional.

Nuestro recorrido consta de cuatro momentos. En primer lugar, haremos una breve introducción al contexto de producción de la obra y mostraremos cómo esta es pasible de ser enmarcada en la más amplia reflexión del autor en torno al imperialismo. A continuación, precederemos a recuperar el ordenamiento jurídico que regulaba la condición legal de los metecos en la Atenas de Jenofonte y la situación demográfica que estaba padeciendo la pólis. En el tercer momento, los pasajes de los Póroi consignados serán puestos en diálogo con otras obras del corpus xenophonteum, puntalmente la Constitución de los Lacedemonios y la Ciropedia, las cuales nos brindarán herramientas necesarias para comprender acabadamente algunas de las propuestas sugeridas. Por último, mostraremos cómo la operación por la cual los metecos son incluidos en su reflexión económica de la pólis es el resultado de un proceso de objetivación estructurado a partir del concepto de "utilidad".

\section{La Propuesta de los Póroi y el “antiIMPerialismo” JenOFOnTeO}

Los Póroi es considerado el último escrito de Jenofonte, datado normalmente en el 355 a.C., inmediatamente luego del fin de la denominada Guerra Social, que enfrentó a Atenas contra Quíos, Rodas, 
Cos y Bizancio,sus antiguos aliados en la Segunda Liga Ateniense. ${ }^{2}$ Desde el inicio, dicha obra advierte sobre cómo el pasado éxito de la política imperialista ateniense había llevado a "los gobernantes" (oi $\pi p \circ \sigma \tau \dot{\alpha} \tau \alpha l)$ a la errada creencia de que la única forma de asegurar el bienestar económico "del pueblo" ( $\tau 0 \tilde{v}$ $\pi \lambda \dot{\eta} \theta$ ovs) era drenando injustamente los recursos de otras póleis sometidas a su égida. Por ende, lo que intentará hacer los Póroi es desandar esta falsa dicotomía y encontrar un mismo remedio tanto para "su

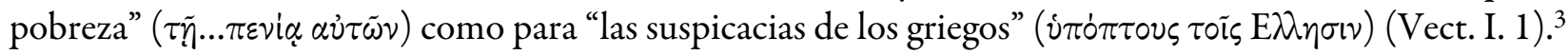

Este "antiimperialismo" que enmarca el comienzo del opúsculo no se restringe solo al caso ateniense, sino que es una temática que cruza gran parte de la reflexión política jenofontea. Mucho se ha discutido sobre su carácter y extensión, siendo las Helénicas y la Ciropedia las fuentes predilectas sobre la cuales se han basado aquellos que ven en su pensamiento una condena del imperialismo genérica y carente de matices. ${ }^{4}$ Pero el resto del corpus también nos provee de pasajes sugerentes en relación con esta temática, como sucede en el problemático capítulo XIV de la Constitución de los Lacedemonios, ${ }^{5}$ donde se argumenta que el principal factor que llevó al abandono de las leyes de Licurgo, de su economía autárquica y propició la decadencia espartana, fue el querer dominar otras póleis:

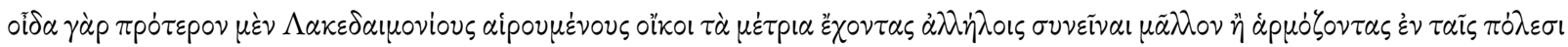

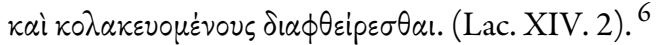

Sé que antes los espartanos preferían convivir entre ellos en su patria con mesuradas posesiones más que corromperse y ser adulados como gobernantes de otras ciudades.

Las opiniones, por el contrario, no han sido del todo pacíficas en torno a los Póroi. Frente a aquellos que lo interpretan como una completa renuncia al imperialismo (Gauthier, 2010, p. 135) o un llamado a adoptar una política aislacionista (Dillery, 1993), otros lo han leído como una exhortación a que Atenas asumiera un liderazgo "justo" y "real" -entendiéndose por oposición al "tiránico" que ejercía con anterioridad- sobre la Hélade (Farrell, 2016). Incluso ha habido quienes lo consideran un programa destinado a fundar el tercer imperio ateniense (Lewis, 2009, p. 378). Si bien el objetivo de este trabajo no es alegar a favor de ninguna de estas interpretaciones, lo que nos interesa enfatizar es que la propuesta de Jenofonte está lejos de ser catalogada como un pacifismo idealista e ingenuo. La hipótesis de una eventual guerra está siempre presente en la obra y Jenofonte advierte que Atenas debe estar preparada para ello (Vect. IV.41-52). Lo que se rechaza es el extremo contrario, igualmente ingenuo, de que la guerra sea el único camino disponible para alcanzar una prosperidad económica estable y duradera (Farrell, 2016, p. 343).

Ahora bien, al reconocer que abandonar la anterior política implicaría dejar de lado la principal fuente de ingresos con la cual Atenas había logrado mantener al dêmos fuera de la pobreza, los Póroi estará estructurado en encontrar nuevas fuentes de ingresos que no pongan en riesgo la diplomacia ateniense. A tal efecto, lo primero que hará Jenofonte será exponer brevemente las ventajosas “condiciones naturales del Ática" ( $\tau \grave{\eta} \nu$

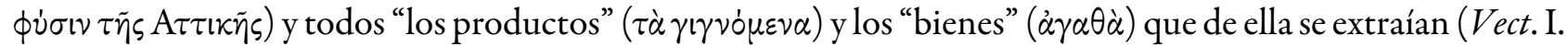
2-8). Pero inmediatamente cambiará de temática, y se abocará a lo que resulta de interés particular en nuestro trabajo: la cuestión poblacional en general, y puntualmente, la situación de los metecos.

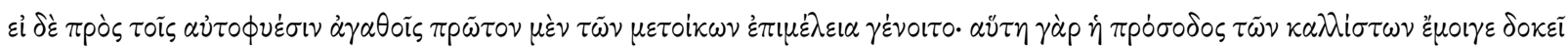

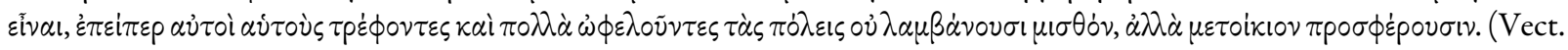
II. 1). ${ }^{7}$

Pero ojalá que, además de los bienes naturales, en primer lugar, por cierto, se prestara cuidado a los metecos. Pues, me parece que este es el ingreso de más valor, ya que ellos mismos se mantienen y, siendo muy útiles a las ciudades, no reciben un sueldo, sino que pagan un impuesto especial.

Lo que más llama la atención de esta exhortación es que, si los metecos están siendo emplazados en la misma balanza que "los bienes naturales", es porque estamos comparando cosas que pertenecen a la misma categoría. Así lo entenderá también Lewis (2009) en su célebre estudio económico de los Póroi, quien considera que Jenofonte nos presenta a los metecos como el "activo" (asset) y el "recurso" (resource) más 
importante de Atenas (p. 374). Es decir, los metecos presentarán una doble naturaleza; no serán solamente sujetos productores de otros bienes en tanto aportantes de una tasa especial, sino que devendrán objetos dentro del cálculo económico que signará su propuesta.

\section{MARCO NORMATIVO Y DEMOGRAFÍA ATENIENSE}

Para lograr una comprensión más consistente del planteo ofrecido por Jenofonte, deviene imperioso realizar un análisis del marco normativo ateniense que regulaba la condición jurídica de los metecos. A su vez, es crucial intentar aproximarnos a la situación demográfica de la pólis al momento de la redacción de la obra.

Es normalmente aceptado que la condición de meteco recaía principalmente sobre aquellos extranjeros que se habían trasladado a Atenas y habían obtenido el derecho de residencia en virtud de la inscripción en un dêmos bajo el patrocinio de un prostátes. ${ }^{8}$ Es importante mencionar que la inclusión en dicha categoría no era optativa, ${ }^{9}$ sino que era una obligación que comenzaba una vez se excedía el límite temporal que había establecido Atenas previo a que se tornara exigible el pago del metoikion. ${ }^{10}$ Las intenciones personales que cada extranjero tuviera, las cuales afloran en algunos estudios bajo la fórmula "sans esprit de retour" (Clerc 1893, p. 12), parecen haber sido intrascendentes desde la perspectiva de la pólis, la cual habría diseñado aquella "maquinaria pragmática" para su determinación (Whitehead, 1977, p. 9). Ahora bien, esta primera aproximación se enfrenta a dos escollos. En primer lugar, hasta finales del siglo III a.C. no contamos con ninguna fuente que nos provea de una definición positiva de dicho concepto. ${ }^{11}$ En segundo lugar, no resulta claro hasta qué punto dicha categoría englobaba a otros grupos poblacionales aparte del mencionado, como es el caso de los esclavos libertos. ${ }^{12}$

Con respecto a su situación jurídica una vez establecidos en Atenas, los metecos carecían de derechos políticos, es decir, no podían ejercer cargos públicos, ni integrar jurados o ser miembros de la Boulé, o de la Ekklesía. ${ }^{13}$ Desde una perspectiva económica, no se les permitía adquirir tierras o inmuebles dentro del Ática, salvo autorización expresa (énktesis), ${ }^{14}$ y a su vez, debían pagar el impuesto ya mencionado: el metoíkion. En el ámbito jurisdiccional, la situación revestía de cierta complejidad. Había acciones que solo los ciudadanos podían presentar, como la graphè hýbreos o la graphbèxenías, y ante una demanda entablada en su contra por un ciudadano ateniense, este último podía solicitar una serie de garantías (engyetaí) para que el extranjero no abandonase el Ática con el fin de escapar de la jurisdicción ateniense. ${ }^{15}$ A su vez, existía una serie de órganos jurisdiccionales especiales para tratar los casos que involucrasen a extranjeros. Por ejemplo, era el polemarco quien resolvía un caso presentado por un meteco. ${ }^{16}$ Por último, estas limitaciones a sus derechos no los eximía de la obligación principal de servir en el ejército.

Para comprender el planteo de Jenofonte en los Póroi, es preciso advertir que la forma en la que la pólis concibió a los metecos en relación con su integración a la sociedad ateniense no fue siempre idéntica. Si durante el siglo V a.C., puntualmente previo a que la Guerra del Peloponeso comenzara a causar estragos, los metecos eran vistos como integrantes fuertemente incorporados a la vida comunitaria de Atenas, ${ }^{17}$ a partir del siglo IV a.C. -inestabilidad económica y política de por medio- y ante el arribo de extranjeros a Atenas en busca de refugio provisorio, los límites que separaban a los metecos de los extranjeros no-residentes comenzaron a difuminarse progresivamente. ${ }^{18}$

Con respecto a la situación demográfica, si bien no podemos hablar de datos certeros, Atenas venía sufriendo un marcado descenso poblacional producto de las sucesivas guerras en las que se había involucrado, el efecto de brotes epidémicos y de determinadas políticas directamente hostiles hacia los extranjeros. ${ }^{19}$ Sobre esto último nos detendremos especialmente. Unas décadas previas a la redacción de los Póroi, el mismo Jenofonte nos informa en sus Helénicas que el gobierno de los Treinta Tiranos (404 a.C.), con la excusa de 
recaudar dinero para pagar la guarnición espartana apostada en la ciudad, aunque principalmente impulsados por su rechazo ideológico hacia la población meteca, decidió: ${ }^{20}$

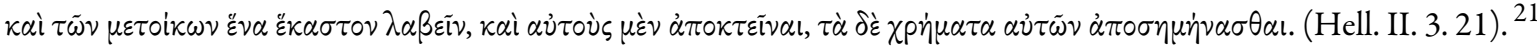

(...) Que cada uno detuviera a un meteco, le diese muerte y confiscara sus riquezas.

Es presumible pensar que aquellos metecos que tuvieron la oportunidad hubieran abandonado Atenas. Pero incluso una vez restaurada la democracia y con la vuelta de varios de los metecos expulsados, tanto Whitehead (1977, p. 259) como Clerc (1893, pp. 375-376) sugieren que la población meteca nunca retornó a los picos alcanzados en el siglo $\mathrm{V}$ a.C. Pruebas de esto pueden ser encontradas en el mismo Póroi:

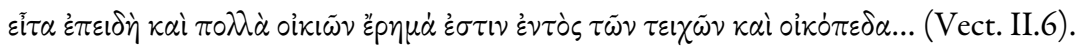

En consecuencia, ya que también muchas de las casas y terrenos dentro de los muros están vacíos (...)

Impresión que también es compartida por Isócrates, aunque expresada de forma más alarmante:

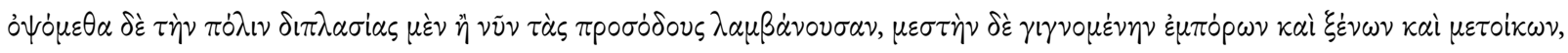
$\tilde{\omega} \nu \nu \tilde{\nu} \nu \dot{\varepsilon} p \dot{\eta} \mu \eta$ $\kappa a \theta \dot{\varepsilon} \sigma \tau \eta \kappa \varepsilon v$. (Isoc. VIII. 21). ${ }^{22}$

Veríamos que la ciudad recibiría el doble de ingresos que en la actualidad, que estaría llena de comerciantes, extranjeros y metecos, de los que ahora está vacía.

Que tanto Jenofonte como Isócrates, escribiendo en el mismo momento, hayan utilizado el adjetivo éremos para describir la situación demográfica ateniense da cuenta de su gravedad y de la ostensible diferencia con períodos anteriores. Con este telón de fondo, en consecuencia, no resulta llamativo que Jenofonte decidiese enfocarse de lleno a la cuestión poblacional para revertir la escasez de ingresos que padecía la ciudad.

\section{LA CUESTIÓN METECA Y EL PROBLEMA DE LA POBLACión EN JENOFONTE}

El hecho de que el filósofo ateniense haya rápidamente virado el interés de los recursos naturales hacia el tratamiento de la población meteca no solo responde a la situación particular de Atenas, sino que es una constante que aparece reiteradas veces en su reflexión política. Dos pasajes puntuales, uno de la Constitución de los Lacedemonios y otro de la Ciropedia, son sumamente ilustrativos y permiten comprender mejor el planteo de los Póroi. En el primer caso, el contraste permite focalizar en el debate respecto de la calidad y cantidad de los habitantes; del segundo texto, nos interesará recuperar la lógica objetivante que opera con relación al grupo de los metecos.

En relación entonces con la Constitución de los Lacedemonios, debe advertirse que la cuestión poblacional reviste allí de una importancia central; la presencia humana en la pólis puede considerarse incluso uno de los ejes articulares del discurso, como puede desprenderse de su mismo inicio:

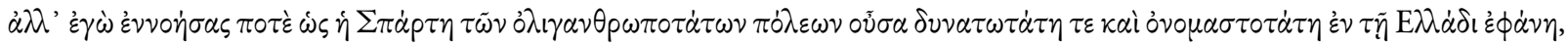

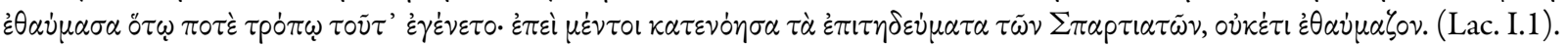
En efecto, al comprender un día que Esparta, estando entre las ciudades con menos hombres, fue claramente la más poderosa y afamada, me admiré del modo en que llegó a suceder esto. No obstante, cuando estudié detenidamente las costumbres de los espartanos dejé de sorprenderme.

La sorpresa del narrador es posiblemente el aspecto determinante del pasaje. Estaría confirmando que la regla general al momento de determinar la prosperidad de una pólis estaba ligada al elemento demográfico, y dentro de este, a su aspecto cuantitativo. Es decir, lo lógico hubiese sido suponer que, si Esparta estaba escasamente poblada, no hubiese habido forma de que se convirtiera en la pólis "más poderosa y afamada". Ahora bien, como se explicará en el devenir de la obra -y esto es lo que debemos enfatizar- el hecho de que Esparta fuese una excepción a dicha regla, no radicaba en que había desatendido la cuestión poblacional, sino que había decidido otorgar prioridad a su aspecto cualitativo por sobre el cuantitativo. Justamente, el 
narrador señala que su sorpresa cesó una vez que estudió detenidamente sus costumbres, las cuales regulaban principalmente ciertos ámbitos como la procreación (I. 3-10), la educación de los jóvenes (II. 1 - IV. 7) y el modo de vida en un sentido general (V. 1 - X. 8). Es decir, toda la maquinaria estatal espartana estaba estructurada a los efectos de garantizar la calidad de su población.

Pero ¿̇cómo se relaciona de modo más específico este dato con los Póroi, más allá de que sirva en general para confirmar el interés de Jenofonte respecto del elemento poblacional? Desde ya que, si analizamos las políticas puntuales llevadas adelante por Esparta bajo el régimen de Licurgo en torno al manejo de su población, estas difieren en gran medida de las que el mismo Jenofonte estaba recomendando para Atenas. Mientras que Jenofonte llama a Atenas a abrazar una política que atrajera inmigrantes, las políticas espartanas estaban enfocadas solamente en sus ciudadanos, lo que acarreaba que los extranjeros fuesen incluso vistos como un elemento contaminante. ${ }^{23}$ Evidentemente, las respectivas medidas tenían como destinatarios distintos grupos poblacionales -la primera se dirigía a los metecos, mientras que la segunda a los ciudadanos- de modo que ver una inspiración lacedemonia directa en los Póroi sería engañoso.

Ahora bien, lo que quisiéramos remarcar es que la importancia otorgada a la cuestión "cualitativa" de la población no resulta exclusiva de Esparta. En los Póroi de ninguna forma se aboga por una inmigración descontrolada y carente de criterio al momento de otorgar beneficios a los metecos. Esto se deja entrever en dos de las reformas jurídicas que propone para ellos.

En primer lugar, el texto sugiere eximirlos de la obligación de formar parte del contingente de hoplitas, aunque al mismo tiempo alienta a que se los incluya en la caballería (Vect. II. 5), una unidad honorífica asociada tradicionalmente a los ciudadanos aristocráticos. ${ }^{24}$

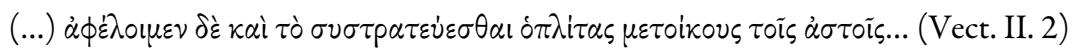

(...) y también eximamos a los metecos de servir en campaña como hoplitas junto a los ciudadanos.

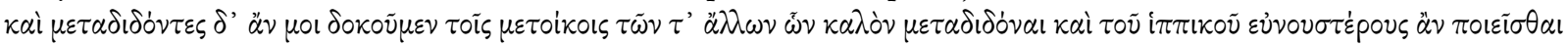

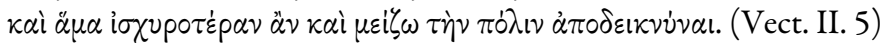

Y si hiciéramos partícipes a los metecos de la caballería y de otros privilegios que es noble dar, me parece que los volveríamos más dispuestos y, al mismo tiempo, mostraríamos a la ciudad más fuerte y más importante.

Muchos -donde nos incluimos- han visto en esta decisión una hábil política orientada a atraer extranjeros con un alto nivel de ingresos, en tanto deberían estar en condiciones de costearse la manutención de un caballo. $^{25}$

En segundo lugar, otra importante reforma sugerida por Jenofonte es la de otorgarles el derecho de énktesis oikías, es decir, el permitir que los metecos adquirieran casas y terrenos dentro de las murallas de Atenas: ${ }^{26}$

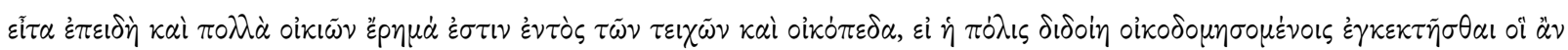

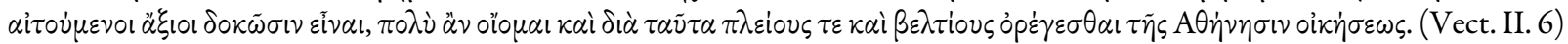
En consecuencia, ya que también muchas de las casas y terrenos dentro de los muros están vacíos, si la ciudad permitiera adquirirlos a los que van a construir -siempre que habiéndolo solicitado parecieran que son dignos- creo fuertemente que, por esto, más y mejores < metecos $>$ desearían residir en Atenas.

Una lectura detenida del pasaje, el cual ya había aparecido parcialmente, evidencia que Jenofonte establece sutiles condicionantes a este beneficio. En primer lugar, el filósofo es muy preciso en circunscribir la presente medida a los inmuebles ubicados en el núcleo urbano de la ciudad, excluyendo la posibilidad de que los metecos adquirieran tierras rurales. En segundo lugar, Jenofonte estaría sugiriendo, aunque sin desarrollarlo en profundidad, la instauración de un procedimiento de dos fases. Por medio de la utilización del participio del verbo $\alpha i \tau \dot{\varepsilon} \omega$, deducimos que serían los metecos quienes deberían, en primer lugar, solicitar dicho beneficio. No estamos ante una política de entrega de tierras masiva impuesta por la pólis, sino ante otorgamientos individuales y a demanda. Pero incluso así, no bastaría meramente con exigir el beneficio. Habría una

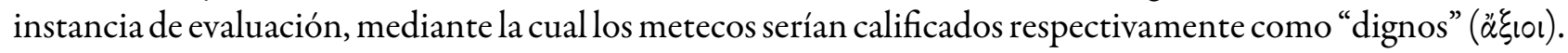
Por último, Jenofonte explicita que la medida no está dirigida solamente a atraer "más" ( $\pi \lambda \varepsilon$ हiovs) personas, lo 
que daría cuenta del elemento cuantitativo de su propuesta, sino también "mejores" ( $\beta \varepsilon \lambda \tau i$ iovs), retomando el carácter cualitativo que vimos en la anterior propuesta relacionada con la inclusión de los metecos a la caballería. Parecería que Jenofonte estaría buscando atraer aquello que Clerc (1893) denominó como una "aristocracia meteca" (p. 440).

La comparación con la Ciropedia, por su parte, nos permite reflexionar sobre el proceso de objetivación que permitió conceptualizar a los metecos como bienes y así dar cuenta de su anclaje filosófico. Promediando la mitad del Libro IV de la Ciropedia, una vez que las caballerías meda e hircania (aliadas de los persas) han regresado al campamento luego de una exitosa persecución de las tropas asirias, Ciro se muestra deseoso por saber si el territorio que habían recorrido, aparte de tener bienes naturales, "estaba habitado" (oikoĩ $\tau$ ) (Cyr. 4.4.4), dado que:

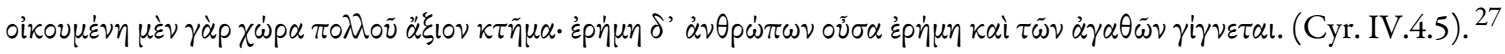

(...) Un país habitado es una adquisición de gran valor, mientras que un país vacío de hombres se acaba convirtiendo en un país vacío de bienes.

En este pasaje advertimos que reaparece el gesto que vimos en los Póroi, en el que los metecos, al ser pasados por un tamiz objetivante, eran puestos en la misma balanza que los bienes naturales. La diferencia se encuentra en que ahora dicha asociación es explicitada al máximo y es aplicada de una forma más amplia, referida a la totalidad de la población y no sólo a un grupo determinado. Por último, que el adjetivo elegido nuevamente sea éremos nos confirma que aquel temor de Ciro sobre la eventual disminución de la población es el que terminó materializándose en la Atenas de los Póroi.

Haber incorporado a nuestro análisis la Constitución de los Lacedemonios y la Ciropedia nos permite comprender cómo las propuestas de Jenofonte en los Póroi no son elaboraciones ad hoc desvinculadas del resto de su corpus y sin un sustento filosófico. El problema poblacional emerge claramente en sus obras, incluso en contextos que exceden ampliamente al ateniense, e incluso al heleno. También nos permiten advertir ese doble carácter, cuantitativo y cualitativo, con el que Jenofonte concibe a la población de un determinado territorio y, más importante aún, nos ayuda a dar un paso más en la comprensión de aquella operación objetivante que estaría realizando al momento de incorporar a los metecos a su reflexión sobre los ingresos de Atenas.

\section{LOS METECOS FRENTE A LOS ESCLAVOS Y CIUDADANOS}

Por el momento, nuestro análisis de los Póroi ha dejado de lado otros grupos poblacionales que también son mencionados allí: los esclavos y los ciudadanos. Al compararlos con los metecos, emergen dos preguntas imposibles de evadir: ¿hasta qué punto nuestra interpretación sobre la forma en la que Jenofonte entiende a los metecos en los Póroi no termina por igualarlos a la población esclava? ¿Por qué Jenofonte considera a los metecos de esta forma, sin atribuirle a los ciudadanos atenienses igual característica?

En primer lugar, debemos señalar que el aumento de la población de esclavos es otra de las reformas cruciales sugeridas por Jenofonte. En Vect. IV. 17-32 propondrá un esquema sumamente complejo donde la pólis misma es la que adquiere esclavos públicos que luego serían destinados a la extracción de plata en las minas de Laurión. ${ }^{28}$ Resulta notable que al referirse a los metecos, en ningún momento se mencionen cifras precisas ni se plantee una meta puntual que deba ser alcanzada; en cambio, al introducirnos a la cuestión esclava, llama considerablemente la atención la exactitud de Jenofonte: ${ }^{29}$ se necesitaría que la pólis adquiriese tres esclavos por cada ciudadano (Vect. IV. 17) y esto lo lograría a través de un procedimiento escalonado, comenzando con una compra inicial de mil doscientos y utilizando las ganancias producidas para futuras adquisiciones de contingentes de diez mil esclavos (Vect. IV. 23-24). Sobre la base de este testimonio, Gauthier (2010, pp. 116 y 121) especula que el programa jonofonteo demandaría la incorporación de entre sesenta y noventa mil esclavos públicos. 
Esto nos muestra que la objetivación a la que hicimos mención no se refiere a un cambio en la situación jurídica de los respectivos grupos poblacionales. Todo lo contrario, desde dicha perspectiva, las reformas de Jenofonte tenderían a reafirmar la diferencia legal de los metecos en relación con los esclavos. Como se advirtió, mientras que las políticas orientadas a aumentar la población meteca apelaban a generar los incentivos suficientes como para atraerlos libremente a Atenas, el aumento de la población esclava se presenta como una incorporación compulsiva de estos. ${ }^{30}$ Ahora bien, es innegable que desde la perspectiva estrictamente económica ambos grupos poblacionales gozarán de igual importancia en el aumento de los ingresos de Atenas y serán pasibles de ser entendidos como bienes dentro de sus estimaciones, con prescindencia de que, conforme al derecho ático, solo los metecos fuesen considerados sujetos de derecho. ${ }^{31}$

El panorama descripto termina por esclarecerse si retomamos brevemente el pasaje de la Ciropedia ya citado. Del planteo de Ciro advertimos que el valor otorgado a la población conquistada no surge, como alguien podría objetar, de que estos serían inmediatamente esclavizados. Todo lo contrario, Ciro les promete que si se mantienen sumisos:

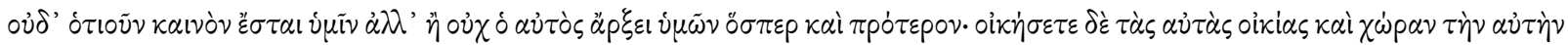

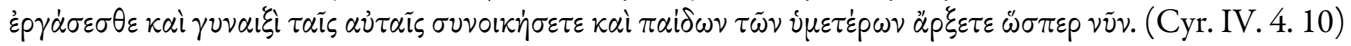

No sufriréis ningún cambio, excepto que quien os gobernará no va a ser la misma persona que antes. Habitaréis las mismas casas, trabajaréis la misma tierra, conviviréis con las mismas mujeres y tendréis autoridad sobre vuestros hijos, como ahora.

Con respecto a la segunda pregunta, la contraposición entre metecos y ciudadanos no es tan explícita como la anterior, pero se mantiene latente durante toda la obra. La diferencia se estructura en torno a que los

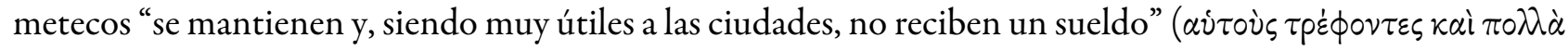
$\dot{\omega} \phi \varepsilon \lambda \circ \tilde{v} \nu \tau \varepsilon \varsigma \tau \dot{\alpha} \varsigma \pi \dot{\partial} \lambda \varepsilon เ \varsigma$ ov่ $\lambda \alpha \mu \beta \dot{\alpha} \nu 0 v \sigma \iota \mu \iota \sigma \theta \dot{v} v, V e c t$. II.1), mientras que los ciudadanos atenienses -por lo menos en un sistema democrático- dependen de recursos externos al percibir un salario del erario público. ${ }^{32}$

El hecho de que Jenofonte haya utilizado el participio del verbo $\omega \dot{\phi} \varepsilon \lambda \dot{\varepsilon} \omega$ no es casual, dado que justamente sobre la idea de "utilidad" parecería estar construido el mecanismo teórico que le permite objetivar a un determinado grupo poblacional para así concebirlo en clave económica. ${ }^{33}$

\section{De la Pólis al ô̂Kos: los PóRoI a la luz de la teoría de la POSESIón del eConómiCo}

El mecanismo recién descripto, referido al proceso de objetivación fundado en la idea de "utilidad", es el que estaría operando de forma incluso más explícita en el comienzo del Económico, donde Jenofonte nos presenta lo podría llamarse su "teoría de la posesión". ${ }^{34}$ Dicha obra comienza con una discusión entre Sócrates y Critóbulo en torno a la posibilidad de considerar la oikonomía ("administración de la hacienda") una epistéme (“ciencia”). Ante la pregunta de Sócrates sobre cómo definir un oîkos, Critóbulo responde:

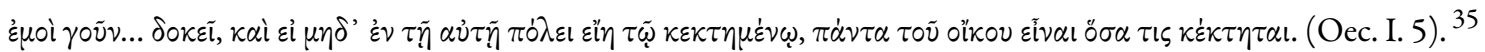

$\mathrm{Al}$ menos en mi opinión (...) todo lo que uno posee, aunque ni siquiera esté en la misma ciudad que el poseedor, forma parte de la hacienda.

A continuación, se presenta la primera definición de la "posesión" (ktêma):

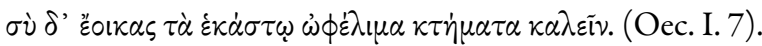

Me da la impresión de que tú llamas posesión a lo que es útil para cada uno.

Lo que quisiera remarcar es que, gracias a la incorporación del concepto de "posesión” y su anclaje a la noción de "utilidad" es que se abre la puerta para que Sócrates y Critóbulo comiencen a considerar que las "posesiones" (ktémata) -o también indistintamente las "propiedades" (khrémata)-de un sujeto no se reducen a los objetos, sino que engloban a distintas categorías de personas, como a los amigos: 


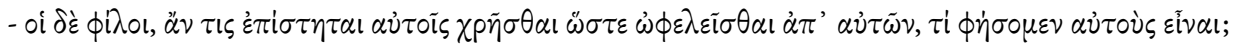

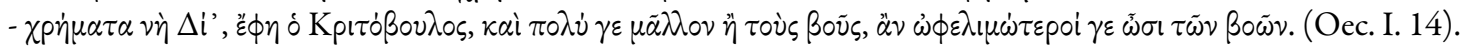

Sócrates: En cuanto a los amigos, si uno sabe usarlos para sacar utilidad de su amistad, iqué diremos que son?

Critóbulo: ¡Propiedades, por Zeus!, dijo Critóbulo, y con mucha mayor razón que los bueyes, puesto que son más útiles que ellos.

Incluso, en el pasaje, se llega al extremo de considerar que corresponde incorporar a los enemigos dentro de aquello que se posee:

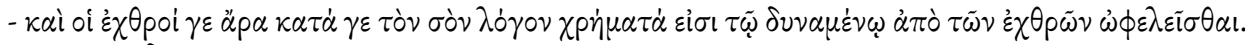

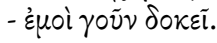

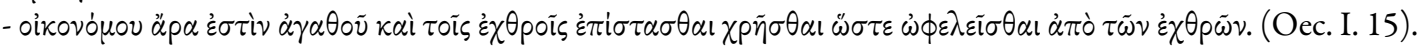

Sócrates: Entonces, según tu propio razonamiento, los enemigos son también propiedades para el que sea capaz de sacar utilidad de ellos.

Critóbulo: Así me lo parece.

Sócrates: Luego, es propio de un buen administrador saber usar también a los enemigos para obtener utilidad de ellos.

Todo indicaría que estamos ante el mismo mecanismo que advertimos en el Póroi con relación a los metecos, ya que nuevamente la idea de "utilidad" se nos presenta como la piedra de toque que permitirá que diferentes personas sean susceptibles de ser incorporadas a un cálculo estrictamente económico. ${ }^{36}$

Desde ya, reconocemos que una posible crítica a la aplicación de esta "teoría de la posesión" desarrollada en el Económico para explicar la lógica que estaría operando en los Póroi bien podría ser que, en el primer texto, Jenofonte limite su pensamiento estrictamente al ámbito del ô̂kos y no al de la pólis, a diferencia de lo que sucede en la segunda obra. A su vez, mientras que en el Económico los conceptos utilizados son los de ktémata ("posesiones") y khrémata ("propiedades"), en los Póroi la discusión gira en torno de agathá ("bienes”). De todas formas, esta diferencia terminológica termina por diluirse cuando recuperamos el famoso criterio jenofonteo en torno a la distinción entre el ồkos y la pólis. Para el filósofo ateniense lo que separa a uno de la otra es una cuestión meramente cuantitativa, es decir, la cantidad de individuos sobre los que se debe mandar. La pólis no es un fenómeno social de carácter excepcional ni el ideal hacia el cual toda agrupación de individuos necesariamente deba tender. Por consiguiente, lo que vale para la pólis, vale también para el ồkos, y viceversa. ${ }^{37}$ Es tal vez en Memorabilia donde Jenofonte más enfatiza este criterio. Allí Sócrates sostiene que:

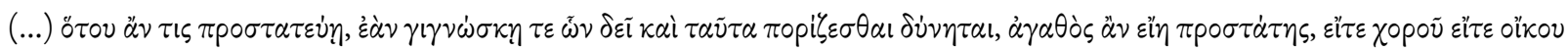

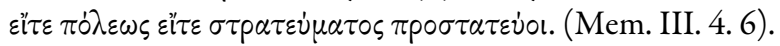

(...) Quien quiera que mande, si conoce lo que tiene que saber y es capaz de poner los medios, será un buen jefe tanto si tiene que mandar un coro, una casa, una ciudad o una guerra.

Y concluye que:

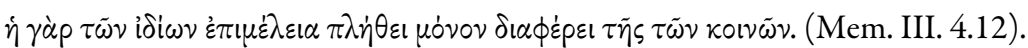

El cuidado de los negocios privados solo se diferencia del de los comunes en su número.

De esta forma, al interpretar el proceso de objetivación señalado en los Póroi a la luz la teoría de la posesión del Económico y al presentar el paralelismo que Jenofonte concibe entre la esfera del ốkos y la pólis, es posible advertir cómo la forma en la que Jenofonte concibe a la población meteca y las reformas que sugiere implementar específicamente en Atenas se encuentran sustentadas en consistentes bases filosóficas.

\section{Conclusión}

Los Póroi ha sido una obra tradicionalmente relegada dentro del estudio del corpus xenophonteum; situación marginal que se vio agravada al haber sido Jenofonte excluido por mucho tiempo del canon filosófico, incluso recibiendo el mote de "socrático mayor". Yendo en contra de dicha corriente, lo que se ha 
pretendido hacer en este artículo fue mostrar cómo, en uno de sus trabajos de menor extensión y a priori con una finalidad eminentemente práctica y circunscripta a una problemática local, sus propuestas de reformas legales y económicas se encuentran fundadas en claros presupuestos filosóficos.

Como punto de partida, consideramos que es posible encuadrar los Póroi dentro de la compleja reflexión jenofontea sobre los inconvenientes inherentes a todo proyecto imperialista. Desde allí, nuestra investigación procuró explicar el interés que Jenofonte otorga a la cuestión poblacional, y puntalmente, a la población meteca, a la luz de dos importantes factores. En primer lugar, si bien no contamos con datos certeros, es innegable que Atenas venía sufriendo un marcado descenso demográfico que impactaba de lleno en su recaudación y flujo comercial, por lo que era esperable que en este contexto los Póroi le otorgasen especial consideración. En segundo lugar, es notable que dicha importancia reaparezca en otras obras del filósofo que no tienen por objeto referirse a la situación ateniense, como es el caso de la Constitución de los Lacedemonios, ni incluso al caso puntual de lo que acontece en Grecia, como es el caso de la Ciropedia. Ambos textos, al ser puestos en diálogo con los Póroi, nos permiten comprender con mayor nitidez las reformas que Jenofonte solo enuncia superficialmente en este último trabajo. Como pudo verse, la inclusión de los metecos a la caballería y los condicionantes que establece a la entrega de tierras para la construcción de casas dan cuenta de la importancia atribuida a la calidad de los metecos que se buscaban atraer. ${ }^{38}$

No obstante ello, el corazón de la reflexión de Jenofonte sobre los metecos no debe buscarse en la finalidad perseguida por cada reforma ni en los efectos que aquellas tendrían de ser adoptadas, sino en la forma en la que el filósofo decide en un primer momento incorporar a los metecos a sus cálculos económicos, poniéndolos en la misma balanza que cualquier otro tipo de bien. Consideramos, en definitiva, que allí estaría operando un proceso de objetivación calibrado por el concepto de "utilidad", el cual hunde sus raíces en la teoría de la posesión expuesta al comienzo del Económico.

\section{REFERENCIAS}

Austin, M. y Vidal-Naquet, P. (1972). Économies et sociétés en Grèce ancienne. Paris: Armand Colin.

Akrigg, B. (2019). Population and Economy in Classical Athens. Cambridge: Cambridge University Press.

Azoulay, V. (2009). Cyrus, disciple de Socrate? Public et privé dans l'œuvre de Xénophon. Études platoniciennes, 6, 153-173.

Azoulay, V. (2018). Xenophon and the Graces of Power: A Greek Guide to Political Manipulation. Swansea: Classical Press of Wales.

Balot, R. (2006). Greek Political Thought. Oxford: Blackwell.

Bodei Giglioni G. (Ed.) (1970). Xenophontis De Vectigalibus. Firenze: La Nuova Italia.

Buis, E. J. (2019). El juego de la ley. La poética cómica del derecho en las obras tempranas de Aristófanes (427-414 a.C.). Madrid: Dykinson \& Universidad Carlos III.

Carey, C. (1995). Rape and Adultery in Athenian Law. The Classical Quarterly, 45(2), 407-417.

Clerc, M. (1893). Les métèques athéniens: étude sur la condition légale, la situation morale et le rôle social et économique des étrangers domiciliés à Athènes. Paris: Thorin \& Fils Éditeurs.

Demont, P. (1982). La polysémie d'un verbe grec: $\tau p \dot{\varepsilon} \phi \omega$, "cailler, coaguler, nourrir, élever". Études de littérature ancienne, 2, 111-22.

De Romilly, J. (1954) Les modérés athéniens vers le milieu du IVe siècle: échos et concordances. Revue des Études Grecques, 67, 327-54.

Dillery, J. (1993). Xenophon's "Poroi” and Athenian Imperialism. Historia, 42(1), 1-11.

Dillery, J. (1995). Xenophon and the History of His Times. London: Routledge.

Farrell, C. A. (2016). Xenophon Poroi 5. Securing a 'More Just' Athenian Hegemony. Polis, The Journal for Ancient Greek Political Thought, 33, 331-355. 
Figueira, T. (2003). Xenelasia and Social Control in Classical Sparta. The Classical Quarterly, 53(1), 44-74.

Fisher, N. (1990). The law of hybris in Athens. En P. Cartledge, P. Millett y S. Todd (eds.), Nomos: Essays in Athenian Law, Politics and Society (pp. 123-138). Cambridge: Cambridge University Press.

Fornis, C. (2019). La xenalesia espartana. En F. Marco Simón, F. Pina Polo y J. Remesal Rodríguez (Eds.), Xenofobia $y$ racismo en el mundo antiguo (pp. 85-99). Barcelona: Edicions de la Universitat de Barcelona.

Gauthier, P. (1972). Symbola. Les étrangers et la justice dans les cités grecques. Nancy: Université de Nancy.

Gauthier, P. (1976). Un commentaire historique des Poroi de Xénophon. Paris: Librairie Droz.

Gauthier, P. (2010). Xenophon's Programme in the Poroi. En V.J. Gray (ed.), Xenophon (pp. 113-136). Oxford: Oxford University Press. [= (1984) Le programme de Xénophon dans les Poroi. Revue de Philologie, 58, 181-199.]

Gomme, A. W. (1934). Two Problems of Athenian Citizenship Law. Classical Philology, 29(2), 129-130.

Gray, V.J. (1998). The Framing of Socrates. The Literary Interpretation of Xenophon's Memorabilia. (Hermes Einzelschriften 79). Stuttgart: Franz Steiner-Verlag.

Guntiñas Tuñón, O. (Ed.). (1982). Jenofonte. Helénicas. Madrid: Gredos.

Guzmán Hermida, J. M. (Ed.). (1982). Isócrates. Discursos. Madrid: Gredos.

Hansen, M. H. (1991). The Athenian Democracy in the Age of Demosthenes. Oxford: Blackwell.

Harrison, A. R. W. (1971). The Law of Athens, vol. II. Oxford: Oxford University Press.

Humble, N. (2004). The author, date and purpose of Chapter 14 of the Lakedaimoniôn Politeia. En C. Tuplin (Ed.), Xenophon and His World (pp. 215-228). Stuttgart: Franz Steiner Verlag.

Hunter, V. (2000). Introduction: status distinctions in Athenian law. En V. Hunter y Edmondson, J. (Eds.), Law and Social Status in Classical Athens (pp. 1-29). Oxford: Oxford University Press.

Ismard, P. (2014). The Single Body of the City: Public Slaves and the Question of the Greek State. Annales. Histoire, Sciences Sociales, 69(3), 503-532.

Ismard, P. (2017). Democracy's Slaves: A Political History of Ancient Greece. Cambridge: Harvard University Press.

Johnson, D. (2005). Persians as Centaurs in Xenophon's "Cyropaedia". Transactions of the American Philological Association, 135(1), 177-207.

Kamen, D. (2013). Status in classical Athens. Princeton: Princeton University Press.

Lévy, E. (1988). Métèques et droit de résidence. En Lonis, R. (Ed.), L'étranger dans le monde grec. Actes du colloque organisé par l'Institut d'Études Anciennes (pp. 47-67). Nancy: Université de Nancy.

Lewis, D. (2009) Xenophon's Póroi and the Foundations of Political Economy. Polis, The Journal for Ancient Greek Political Thought, 36, 370-88.

MacDowell, D. M. (1978). The Law in Classical Athens. Ithaca: Cornell University Press.

Mansouri. S. (2011). Athènes vue par ses métèques (Ve-IVe siècle av.J.-C.). Paris: Tallandier.

Manville, P. B. (1990) The origins of citizenship in ancient Athens. Princeton: Princeton University Press.

Marchant, E. C. (Ed.) (1920). Xenophon. Xenophontis opera omnia, vol. 5. Oxford: Clarendon Press.

Mársico, C., Illarraga R. y Marzocca. (Eds.). (2017). Jenofonte/Pseudo Jenofonte: Constitución de los lacedemonios, Constitución de los Atenienses, Hierón. Bernal: Universidad Nacional de Quilmes.

Meyer, E. A. (2010). Metics and the Athenian phialai-inscriptions: A study in Athenian epigraphy and law. Stuttgart: Franz Steiner Verlag.

Morrow, G. R. (1939). Plato's Law of Slavery in its Relation to Greek Law. Urbana: The University of Illinois Press.

Nadon, C. (2001). Xenophon's Prince: Republic and Empire in the Cyropaedia. Berkeley: University of California Press.

Norlin, G. (Ed.) (1929). Isocrates. On the Peace. Areopagiticus. Against the Sophists. Antidosis. Panathenaicus. Cambridge: Harvard University Press.

Osborne, R. (1985). Law in Action in Classical Athens. Journal of Hellenic Studies, 105, 40-58

Partsch, J. (1909). Griechisches Burgschaftsrecht. Leipzig: Teubner. 
Pečirka, J. (1966). The Formula for the Grant of Enktesis in Attic Inscriptions (Acta Universitatis Carolinae Philosophicae et Historica Monographica, xv). Praha: Univerzita Karlova.

Sinclair, R. K. (1991). Democracy and Participation in Athens. Cambridge: Cambridge University Press.

Schmitt-Pantel, P. (1992). La Cité au banquet. Histoire des repas publics dans les cités grecques. Rome: Ecole Française.

Schütrumpf, E. (1982). Xenophons Vorschläge zur Beschaffung von Geldmitteln oder Über die Staatseinkünfte. Darmstadt: Wissenschaftliche Buchgesellschaft.

Tamiolaki, M. (2009). Public et privé dans le dialogue de Socrate avec Aristippe (Xén. Mém. II, 1, 1- 33). Études platoniciennes, 6, 141-151.

Todd, S.C. (1993). The Shape of Athenian Law. Oxford: Clarendon Press.

Tuplin, C. (1993). The Failings of Empire. Stuttgart: Franz Steiner Verlag.

Van Wees, H. (2011). Demetrius and Draco: Athens' property classes and population in and before 317 BC. Journal of Hellenic Studies, 131, 95-114.

Vegas Sansalvador, A. (Ed.) (1987). Jenofonte. Ciropedia. Madrid: Gredos.

Whitehead D. (1977). The ideology of the Athenian metic. Cambridge: Cambridge Philological Society.

Wilamowitz-Möllendorf, U. Von. (1887). Demotika der attischen Metoeken. Hermes, 22, 107-128, 211-259.

Zaragoza, J. (Ed.) (1982). Jenofonte. Recuerdos de Sócrates y diálogos. Madrid: Gredos.

Zelnick-Abramovitz, R. (2005). Not Wholly Free: The Concept of Manumission and the Status of Manumitted Slaves in the Ancient Greek World. Leiden \& Boston MA.

Zelnick-Abramovitz, R. (2009). Freed slaves, their status and state control in Ancient Greece. European Review of History: Revue europeenne d'histoire, 16(3), 303-318.

\section{Notas}

1 Este trabajo es el producto de las investigaciones llevadas a cabo en el marco del Grupo de Trabajo sobre Derecho Griego Arcaico y Clásico y sus Proyecciones (DEGRIAC), a cargo del Prof. Dr. Emiliano J. Buis, como también del GICP "Gobierno y narración en la teoría política antigua: Jenofonte y los socráticos", a cargo del Prof. Dr. Rodrigo Illarraga.

2 Sobre la datación de los Póroi, ver Bodei Giglioni (1970, pp. vii-xiv).

$3 \mathrm{Al}$ considerar que los Póroi tendría este doble objetivo nos encolumnamos en la interpretación de Farrell (2016, p. 322). Por su parte, Bodei Giglioni (1970) considera que el objetivo último sería estimular la economía ateniense. Para Gauthier (1976), Schütrumpf (1982) y Azoulay (2018, pp. 133-138) la pacificación de Atenas y las reformas fiscales serían medios para aumentar la recaudación impositiva y así lograr la autosuficiencia.

4 Ver especialmente Tuplin (1993), Dillery (1995), Johnson (2005), Balot (2006, p. 151), Nadon (2001, p. 163).

5 Sobre los debates en torno a la autoría, datación y propósito de dicho capítulo, ver Humble (2004, pp. 215-228) y Mársico, Illarraga y Marzocca (2017, p. 141, n. 70).

6 Para los pasajes de Jenofonte, la edición de los textos griegos corresponde siempre a la de Marchant (1920). En el caso de la Constitución de los Lacedemonios, seguimos la traducción de Mársico, Illarraga y Marzocca (2017).

7 Las traducciones de los pasajes de los Póroi son nuestras.

8 Nuestra definición se alinea con la canónica interpretación hecha por Whitehead (1977) para quien la condición de meteco era principalmente una carga que recaía sobre aquellos extranjeros que optaban por residir en Atenas y no un privilegio otorgado a ciertos extranjeros, como se sostenía hasta aquel entonces, ver Wilamowitz-Möllendorf (1887, pp. 107-128) y Harrison (1971, p. 189). Este enfoque ha signado los estudios más modernos, de los cuales también abreva la definición enunciada, MacDowell (1978, p. 77), Manville (1990, p. 11), Sinclair (1991, pp. 28-29), Hansen (1991, pp. 116-120) y Todd (1993, p. 195),

9 Sobre el debate en torno a la voluntariedad de la inscripción en un dêmos, ver Lévy (1988) y Hunter (2000, p. 22). Sobre el rol del prostátes, ver Gauthier (1972, p. 133) y Todd (1993, p. 198).

10 El lapso de dicho tiempo es un tema sumamente debatido entre los académicos. Gauthier (1972) sostiene que el lapso se extendía por un mes, fundando su teoría en que el metoikion consistía del pago de doce dracmas (y él supone que sería una por mes) (pp. 108-111). Cf. Lévy (1988, p. 61). Sobre el pago del metoikion, ver Whitehead (1977, pp. 75-76).

11 La más antigua que nos ha llegado es un fragmento de Aristófanes de Bizancio ( $F r .38$ Nauck). Sobre las fuentes que nos permiten bosquejar una definición negativa, la principal proviene de la Política de Aristóteles (1275a23-25).

12 Sobre esta categoría, ver Whitehead (1977, pp. 16-17) y Zelnick-Abramovitz (2005, pp. 308-334; 2009). 
13 Para un análisis completo de las limitaciones políticas de los metecos, ver MacDowell (1978, pp. 75-78) y Manville (1990, p. 11).

14 Esta autorización podía incluir tierras y casas (gês kai oikias) o solo casas (oikias). Al respecto, ver Pečirka (1966) y Whitehead (1977, p. 30), quien cita las siguientes fuentes epigráficas: IG ii ${ }^{2} 351,360,373,505,551,554,786,835$.

15 Para un completo estudio sobre el procedimiento judicial ateniense, ver Harrison (1971). Para la situación particular de los metecos en el procedimiento judicial, Todd (1993, p. 196) y Kamen (2013, pp. 47-50). Sobre la graphé hýbreos, ver Fisher (1990, pp. 123-138), Carey (1995, p. 410) y Buis (2019, pp. 91-92). Sobre la graphè xenías, ver Gomme (1934, pp. 129-130) y Osborne (1985, p. 56). Para un trabajo antiguo, pero sumamente complete de las engyetaí, ver Partsch (1909).

16 Sobre el rol del polemarco, ver Harrison (1971, pp. 193-199), Sinclair (1991, p. 31) y Todd (1993, pp. 195-196).

17 En Acarnienses de Aristófanes (vv. 502-508), representada por primera vez en el 425 a.C., el protagonista, Diceópolis, traza una analogía sobre la relación entre ciudadanos y metecos; sostiene que, si toda la pólis era una cosecha de trigo, los ciudadanos serían la harina, mientras que los metecos el salvado. Esto mostraría cómo, a pesar de su inferioridad social, se los consideraba parte de un mismo todo, en contraste con los extranjeros visitantes, quienes serían el "cascabillo", aquello que se desecha en la cosecha del trigo. Ver Whitehead (1977, p. 39).

18 Sobre este cambio de percepción, ver Clerc (1893, pp. 111-114 y 123-126), Gauthier (1972, pp. 124-126; 1976, pp. 73-74) y Whitehead (1977, pp. 159-160). Como explica Meyer (2010) para el siglo IV a.C. los metecos eran vistos principalmente como pagadores del metoíkion, siendo fuertemente presionados para que cumplieran con dicha obligación.

19 Durante gran parte del siglo IV a.C., se estima que la cantidad de ciudadanos (hombres adultos hijos de ambos padres atenienses) era de alrededor 30.000, elevándose a 120.000 si se incluyen las mujeres y niños. Estas cifras representaban la mitad de los picos alcanzados inmediatamente antes del estallido de la Guerra del Peloponeso (Akrigg, 2019, p. 169). En relación con los metecos, el único dato preciso, aunque ampliamente discutido, es el censo de Demetrio de Falero, citado por Ctesicles y preservado por Ateneo (FrGrHist 245 F1 = Ateneo, 6.103) donde se habla de una proporción de un meteco por cada dos ciudadanos, teniendo en cuenta que para ese momento la adquisición de la ciudadanía se había limitado a los propietarios (Akrigg, 2019, p. 125) y que posiblemente esos ciudadanos excluidos fuesen incluidos en la categoría de metecos (Van Wees, 2011, p. 101-102)

20 Sobre la ideología anti-meteca del gobierno de los treinta tiranos, ver Whitehead (1977, p. 155)

21 Seguimos la traducción de Guntiñas Tuñón (1982), con modificaciones.

22 La edición del texto griego corresponde a Norlin (1929). Seguimos la traducción de Guzmán Hermida (1982), con modificaciones.

23 Sobre si la xenalesía espartana era una política institucionalizada o una reacción esporádica, ver Figueira (2003) y Fornis (2019). Jenofonte tampoco brinda mucha claridad sobre esta institución. En Lac. XIV, 4 se limita a comentar que, bajo

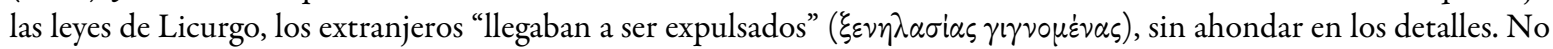
olvidemos que él mismo vivió allí y sus hijos fueron educados en Esparta.

24 Contrario a De Romilly (1954, p. 342) y siguiendo a Azoulay (2018, p. 129) no advertimos una necesaria crítica al uso de mercenarios en el ejército. Su incorporación es incluso recomendada por Jenofonte en Eq. Mag. 9. 3-4.

25 Whitehead (1977, p. 128) y Lewis (2009, p. 375). Sobre la inclusión de los metecos a la caballería, ver Clerc (1893, pp. 56-57), Bodei Giglioni (1970, p. lxv) y Austin y Vidal-Naquet (1972, p. 388, n. 7).

26 Cf. supra. Para Lewis (2009, p. 375) estamos ante una de las reformas más radicales de los Póroi.

27 Para los pasajes de Ciropedia seguimos la traducción de Vegas Sansalvador (1982), con modificaciones.

28 Sobre los eslavos públicos en Grecia, ver Ismard (2017).

29 La combinación de precisión y vaguedad que presenta los Póroi ha sido explicada como una estrategia discursiva de Jenofonte para evitar profundizar en temas políticamente espinosos que impidieran la puesta en marcha de sus reformas (Whithead, 1977, p. 129).

30 Sobre este punto, ver Ismard (2014, p. 514 n. 33). Lewis (2009, p. 384) sugiere que Jenofonte posiblemente esté pensando en esclavos que gozasen de más libertades que las tradicionales.

31 Sobre la situación jurídica de los esclavos, ver Morrow (1939), MacDowell (1978, pp. 79-82) y Todd (1993, pp. 184-194).

32 Sobre la relación entre los conceptos misthós y trophé y las supuestas intenciones antidemocráticas de los Póroi, ver el famoso debate entre Gauthier (1976, pp. 20-32) y Schütrumpf (1982, pp. 65-72), profundizado por Demont (1990, p. 296) y Schmitt-Pantel (1992, p. 174). Para Azoulay (2018, p. 104), Jenofonte nos muestra en el Póroi que la lógica monetaria del misthós no se distancia mucho de la lógica de la kháris, y por ende, un líder hábil podría igualmente utilizarla para su ventaja.

33 Como nos muestra Gray (1998, pp. 113-122) la importancia del “uso” y la "utilidad” en Jenofonte también suele presentarse bajo la palabra khreía.

34 Para otros ejemplos donde el Económico es utilizado como clave interpretativa de los Póroi, consultar Dillery (1993, pp. 7-8), Lewis (2009, pp. 387-388) y Azoulay (2018, p. 136). 
35 Para el Económico y Memorabilia, seguimos la traducción de Zaragoza (1982), con modificaciones.

36 De forma similar Azoulay (2018) sostiene que "By calling for certain categories of the Athenian population to receive different treatment, Xenophon displayed a particular concern for the citizens he deemed truly useful." (p. 137). También Whitehead (1977) cuando, comparando los planteos de Platón y Jenofonte, señala que "Neither has a high regard for the metic as such - only his utility" (p. 135).

37 Sobre las analogías entre la esfera pública y la privada en Jenofonte, ver Tamiolaki (2009) y Azoulay (2009).

38 Acerca de los "buenos" y "malos" metecos en Atenas, ver Mansouri (2011, pp. 73-83). 